\title{
Dietary patterns interact with APOA1/APOC3 polymorphisms to alter the risk of the metabolic syndrome: the Tehran Lipid and Glucose Study
}

\author{
Firoozeh Hosseini-Esfahani ${ }^{1}$, Parvin Mirmiran ${ }^{1,2_{*}}$, Maryam S. Daneshpour ${ }^{3}$, Yadollah Mehrabi $^{4}$, \\ Mehdi Hedayati ${ }^{3}$, Mona Soheilian-Khorzoghi ${ }^{3}$ and Fereidoun Azizi ${ }^{5}$ \\ ${ }^{1}$ Nutrition and Endocrine Research Center, Obesity Research Center, Research Institute for Endocrine Sciences, \\ Shabid Bebeshti University of Medical Sciences, Tehran, Iran \\ ${ }^{2}$ Faculty of Nutrition Sciences and Food Technology, National Nutrition and Food Technology Research Institute, \\ Shabid Beheshti University of Medical Sciences, PO Box 19395 4763, Tehran, Iran \\ ${ }^{3}$ Cellular Molecular and Endocrine Research Center, Research Institute for Endocrine Sciences, \\ Shahid Beheshti University of Medical Sciences, Tehran, Iran \\ ${ }^{4}$ Department of Epidemiology, School of Public Health, Shabid Beheshti University of Medical Sciences, Tehran, Iran \\ ${ }^{5}$ Endocrine Research Center, Research Institute for Endocrine Sciences, Shabid Beheshti University of Medical Sciences, \\ Tebran, Iran
}

(Submitted 31 May 2014 - Final revision received 7 September 2014-Accepted 16 October 2014 - First published online 5 February 2015)

\begin{abstract}
The interaction of genetic and dietary factors, as an area of CVD research, has been explored poorly. The aim of the present study was to examine the interaction of dietary patterns and three genetic variants of $A P O A 1$ and $A P O C 3$, both independently and in combination, relative to the risk of the metabolic syndrome (MetS) in Tehranian adults. In the present matched, nested case-control study, 414 subjects with the MetS and 414 controls were selected from the participants of the Tehran Lipid and Glucose Study. Dietary patterns were determined by factor analysis. APOC3 (rs5128 3238C $>\mathrm{G}$ ) and APOA1 (rs670, $-75 \mathrm{G}>\mathrm{A}$ and rs5069, +83C $>$ T) SNP were genotyped by the conventional PCR followed by the restriction fragment length polymorphism technique. Overall, three major dietary patterns were extracted: healthy dietary pattern (HDP); Western dietary pattern (WDP); fat-sweet dietary pattern (FSDP). The A and T allele carriers of the APOA1 SNP had a greater risk of developing the MetS in the highest quartile of WDP scores (OR $3 \cdot 22,95 \%$ CI $1 \cdot 21,8 \cdot 58$, $\left.P_{\text {interaction }}=0.03\right)$. Compared with other genotype combinations, the combined effect of $A P O C 3 / A P O A 1(\mathrm{CC} / \mathrm{GA}+\mathrm{AA} / \mathrm{CT}+\mathrm{TT})$ genotypes showed a further increase in the risk of the MetS in the highest quartile of WDP scores (OR 1, 2.49, 8.73, 6.32, $\left.P_{\text {trend }}<0.001, P_{\text {interaction }}=0.003\right)$. A significant interaction was found between the quartiles of FSDP scores and the $A P O A 1$ diplotype $(\mathrm{GA}+\mathrm{AA} / \mathrm{CT}+\mathrm{TT})$. OR for these genotype carriers were $1,0.65,0.57$ and $0.22\left(P_{\text {trend }}=0.006\right)$ in the lowest to the highest quartile of FSDP scores when compared with the other combined genotypes $\left(P_{\text {interaction }}=0.03\right)$. Our findings suggest that the WDP and FSDP are associated with $A P O A 1$ and $A P O C 3$ SNP in relation to the risk of the MetS.
\end{abstract}

Key words: Dietary patterns: Genetic polymorphisms: Metabolic syndrome

The metabolic syndrome (MetS) is a common multi-component disorder including abdominal obesity, dyslipidaemia, hypertension and hyperglycaemia, which is known to be associated with an increased risk of CVD and type 2 diabetes ${ }^{(1)}$. Development of the MetS represents an intricate interaction between genetic susceptibilities and environmental exposures ${ }^{(2)}$. Several polymorphisms in $A P O A 1$ and $A P O C 3$ have been described and associated with metabolic diseases. ApoA1, a major component of HDL-cholesterol (HDL-C), is involved in the activation of lecithin cholesterol acyltransferase, which mediates the reverse cholesterol transport from peripheral tissues to the liver, and the inverse relationship between plasma HDL-C levels and CVD has been attributed to this function. ApoC3, a major component of TAG-rich lipoproteins and a minor component of HDL-C, is a non-competitive inhibitor of lipoprotein lipase, suggesting its important role in the catabolism of TAG-rich lipoproteins. The $\mathrm{G}$ allele of the APOC3 3238C > G rs5128 variant has been shown to be associated with increased concentrations

Abbreviations: BP, blood pressure; FSDP, fat-sweet dietary pattern; HDL-C, HDL-cholesterol; HDP, healthy dietary pattern; MetS, metabolic syndrome; PCR-RFLP, PCR followed by restriction fragment length polymorphism; WDP, Western dietary pattern. 
of apoC3, apoB, LDL-cholesterol and plasma TAG, as well as high blood pressure (BP) and hence possibly the development of CHD. Common polymorphisms, such as $-75 \mathrm{G} / \mathrm{A}$ and $+83 \mathrm{C} / \mathrm{T}$, have been identified, respectively, in the promoter region and first intron of the $A P O A 1$ gene located at 11q23. The A allele is known to be associated with higher $A P O A 1$ and HDL-C levels in some populations, whereas the $\mathrm{C}$ allele is associated with higher BMI and waist:hip ratio in type 2 diabetic subjects; however, these associations were not confirmed in other studies ${ }^{(3-6)}$. These inconsistencies could be the result of interactions with environmental factors that modulate the effect of this genetic polymorphism. It is well known that all people are not affected equally by unhealthy lifestyles, with some being more sensitive to deleterious effects than others, heterogeneity which may reflect complex interactions between genetic susceptibilities and environmental factors, particularly $\operatorname{diet}^{(7)}$. To date, most studies have focused on the interaction of dietary fat exposure and genetic background with the risk of the MetS and its phenotypes ${ }^{(8)}$. MetS risk has been shown to increase among GG homozygote individuals for $A P O A 1 \mathrm{rs} 670$, who had high intakes of fat $(>35 \%$ energy) and monounsaturated fat $(>14 \% \text { energy })^{(9)}$. Extracting and defining dietary patterns by evaluating the in-depth associations between nutrients rather than just selecting individual dietary components need to be developed to advance gene-nutrition interaction studies ${ }^{(10,11)}$. Therefore, we aimed to examine the interaction of dietary patterns and three genetic variants of APOA1 (rs670 and rs5069) and APOC3 (rs5128), both independently and in combination, in relation to MetS risk in a nested case-control study of Tehranian adults.

\section{Materials and methods}

\section{Study population}

The present nested case-control study is part of the Tehran Lipid and Glucose Study, an ongoing population-based cohort study conducted to determine the risk factors of noncommunicable diseases in a sample of residents of district no. 13 of Tehran, the capital of Iran. A baseline examination survey was conducted from 1999 to 2001 in 15005 subjects, aged $\geq 3$ years, and follow-up examinations were conducted every 3 years (2002-5, 2006-8 and 2008-11) to update health-related measurements of baseline characteristics and to identify any newly developed diseases ${ }^{(12)}$.

Of the initial group, 11001 and 9807 individuals, aged $\geq 18$ years, participated in baseline and second follow-up surveys, respectively; of which, 5280 individuals were excluded because of having the MetS either at baseline or in the second follow-up survey. In the present study, 503 cases were randomly selected from the participants who developed the MetS at the third ( $n$ 918) and fourth ( $n$ 827) follow-up surveys. After excluding individuals with a history of cardiovascular events, weight loss or gain $>5 \mathrm{~kg}$ in the last 6 months, pregnancy and lactating, or those taking any CVD/anti-coagulant/steroid or hormonal medication ( $n$ 20), 483 cases were included in the present study. Controls were defined as participants with $\leq 1$ MetS components at the time the corresponding case developed the MetS. Each case was individually pair-matched with a control randomly by age ( \pm 5 years) and sex. After excluding cases/controls lacking DNA purification in the range of $1.7<\mathrm{A} 260 / \mathrm{A} 280<2$ ( $n$ 108), and also those whose reported energy intake divided by the predicted energy intake did not qualify for the $3 \mathrm{SD}$ range ( $n$ 30), finally 828 subjects (414 pairs) with the MetS and matched controls remained for the analysis. The study protocol was approved by the ethics committee of the Research Institute for Endocrine Sciences, Shahid Beheshti University of Medical Sciences (SBMU), Tehran, Iran. Written informed consent was obtained from each participant.

\section{Dietary intakes}

Dietary intake was assessed using a valid and reliable 168-item semi-quantitative FFQ, which included a list of foods with standard serving sizes. Validity correlation coefficients ranged from 0.03 (liquid oil) to 0.77 (simple sugars) in men (median 0.44 ) and from 0.12 (snacks) to 0.79 (simple sugars) in women (median $0 \cdot 37)^{(13,14)}$. Based on the macronutrient composition and using the available literature, twenty-five food groups were categorised ${ }^{(15)}$.

\section{Anthropometric and blood pressure measurements}

Weight was measured using digital scales (Seca 707), while the subjects were minimally clothed and without shoes, and recorded to the nearest $100 \mathrm{~g}$. Height was measured using a tape measure (Model 208 Portable Body Meter Measuring Device; Seca) while the subjects were standing, had the shoulders in a normal position and not wearing shoes. BMI was calculated. Waist circumference was measured at the umbilical level using an unstretched tape measure without any pressure to the body surface. Measurements were recorded to the nearest $0 \cdot 1 \mathrm{~cm}$. $\mathrm{BP}$ was measured twice, with at least a $30 \mathrm{~s}$ interval, after the participants rested for 15 min. Finally, the mean BP of the two measurements recorded was reported as the $\mathrm{BP}$ of the subjects.

\section{Physical activity}

Physical activity level was assessed in Tehranian adults using the Persian-translated modifiable activity questionnaire, whose reliability and relative validity was high as reported previously. Data on the frequency and time spent on light, moderate, hard and very hard intensity activities according to the list of common activities of daily life over the past year were obtained. Physical activity levels are expressed as metabolic equivalent-h/week ${ }^{(16-18)}$.

\section{Laboratory assays}

After 12-14 h of overnight fasting, blood samples were drawn into vacutainer tubes between 07.00 and 09.00 hours from all participants in a sitting position ${ }^{(12)}$. The samples were centrifuged within 30-45 min of collection according to standard protocols. All biochemical analyses were carried out at the

\footnotetext{
defined as participants with $\leq 1$ Mets components at the
} 
research laboratory of the Tehran Lipid and Glucose Study on the same day of blood collection, using a Selectra 2 autoanalyser (Vital Scientific). Fasting blood glucose level was measured by the enzymatic colorimetric method using glucose oxidase. TAG level was measured by enzymatic colorimetric tests using TAG kits (Pars Azmoon) and glycerol phosphate oxidase. HDL-C level was measured after precipitation of apoB containing lipoproteins with phosphotungstic acid. Assay performance was monitored once every twenty tests using lipid control serum, Percinorm (reference range) and Percipath (pathological range), wherever applicable (Boehringer Mannheim; catalogue no. 1446070 for Percinorm and 171778 for Percipath). Lipid standard (Cfas, Boehringer Mannheim; catalogue no. 759350) was used to calibrate the Selectra 2 autoanalyser on each day of the laboratory analyses, and all samples were analysed only when internal quality control met the standard criteria. Interand intra-assay CV were both $2 \cdot 2 \%$ for serum glucose and 1.6 and $0.6 \%$ for TAG, respectively.

\section{Genetic analysis}

For genotyping the $A P O A 1 / A P O C 3$ polymorphisms, buffy coats were separated from non-coagulated whole-blood samples and stored at $-70^{\circ} \mathrm{C}$ until processing. Genomic DNA was extracted by the proteinase $\mathrm{K} /$ salting-out method. The PCR followed by the restriction fragment length polymorphism (PCR-RFLP) technique was employed to investigate polymorphisms in gene fragments ${ }^{(19,20)}$.

The PCR was performed using the following primers: APOA1 (rs670, $-75 \mathrm{G}>\mathrm{A}$ and $\mathrm{rs} 5069,+83 \mathrm{C}>\mathrm{T}$ ) (forward: 5'-AGG GAC AGA GCT GAT CCT TGA ACT CTT AAG-3'; reverse: 5'-TTA GGG GCA CCT AGC CCT CAG GAA GAG AGC A-3'); APOC3 (rs5128, C3238> G) (forward: 5'-GGT GAC CGA TGG CTT CAG TT-3'; reverse: 5'-CAGAAG GTG GAT AGA GCG CT- $3^{\prime}$ ). Hybridisation was carried out in a DNA thermal cycler (Corbett Life Science). Amplified DNA was digested with MspI(HpaII) and SacI restriction enzymes (Fermentas) at $37^{\circ} \mathrm{C}$ overnight ${ }^{(21)}$. Fragments were separated by electrophoresis on $2 \%$ agarose gels. DNA fragments were visualised by the gel documentation system (OptiGo; Isogen Life Science); $5 \%$ of all the samples were randomly replicated and genotyped with $\geq 99 \%$ concordance. To confirm the PCRRFLP results, $5 \%$ of the PCR samples were directly sequenced.

\section{Definitions}

The MetS was defined as participants with three or more of the following conditions based on the modified National Cholesterol Education Program/Adult Treatment Panel III ${ }^{(22,23)}$ : (1) $\mathrm{TAG} \geq 1.70 \mathrm{mmol} / 1(\geq 150 \mathrm{mg} / \mathrm{dl})$ or drug treatment; (2) HDL-C $<1.04 \mathrm{mmol} / \mathrm{l}(<40 \mathrm{mg} / \mathrm{dl})$ in men and $<1.30 \mathrm{mmol} / \mathrm{l}(<50 \mathrm{mg} / \mathrm{dl})$ in women or drug treatment; (3) elevated $\mathrm{BP} \geq 130 / 85 \mathrm{mmHg}$ or drug treatment for a previous diagnosis of hypertension; (4) fasting blood glucose $\geq 6.11 \mathrm{mmol} / \mathrm{l}(\geq 110 \mathrm{mg} / \mathrm{dl})$ or drug treatment of hyperglycaemia; (5) waist circumference was coded according to the newly introduced cut-off points for Iranian adults, i.e. $\geq 95 \mathrm{~cm}$ for both sexes ${ }^{(22)}$.
The healthy dietary pattern (HDP) was characterised by a high consumption of different kinds of vegetables, fruits, nuts and seeds, liquid oils, legumes, low-fat dairy products, and whole grains. The Western dietary pattern (WDP) was characterised by a high consumption of fast foods, soft drinks, salty snacks, organ meats, sweets, fish and sweetened fruit juices. The fat-sweet dietary pattern (FSDP) was characterised by a high consumption of sugar, tea and coffee, red meat, solid oils, high-fat dairy products, sweets and green vegetables.

\section{Statistical analyses}

Statistical analyses were performed using the Statistical Package for Social Sciences (version 16.0; SPSS) and STATA (Statistics/Data analysis 12.0). A two-sided $P$ value $<0.05$ was considered significant. Pearson's $\chi^{2}$ statistic was used to test Hardy-Weinberg equilibrium for SNP and to test the differences in percentages using PowerMarker software. Dietary patterns were identified using factor analysis with varimax rotation based on twenty-five regression residual energy-adjusted food groups $(\mathrm{g} / \mathrm{d})$. A total of three factors were retained based on eigenvalues $>1$, the screen plot and interpretability of the factors. Factor loadings represented the correlation between a factor (dietary pattern) and a variable (food group). In the present study, factor loadings below \pm 0.2 are not presented because of the low correlations or low proportions of their food groups explained by the factor, and for easy interpretation of the dietary patterns. Factor scores of each participant were calculated by the sum of multiplying the intake of standardised food groups by their respective factor loadings on each pattern. When the participants had higher scores on a particular factor, they had higher relative adherence to this pattern, compared with others. To compare the characteristics of cases and controls, $\chi^{2}$ and Student's $t$ tests were used for qualitative and quantitative variables, respectively. TAG concentration was logtransformed for statistical analysis. The relationship between characteristics and dietary intakes of the study population across the quartiles of dietary pattern scores was evaluated using the ANCOVA, and the adjusted means were estimated after controlling for age, sex, smoking (current smoker, exsmoker and never smoked), physical activity (low, moderate and high) and education levels ( $\leq 14$ and $>14$ years). Multiplicative interactions between quartiles of dietary pattern scores and polymorphisms (dominant model) in relation to the risk of the MetS were examined in the whole population and by sex, using conditional logistic regression analysis after adjustment for baseline BMI and using the likelihood ratio test, which is a comparison of the likelihood scores of the two models with and without the interaction terms. Conditional logistic regression analysis was used to examine the combined role of quartiles of dietary pattern scores and genotypes of rs5128 (CC/CG + GG), rs670 (GG/GA + AA) and rs5069 $(\mathrm{CC} / \mathrm{CT}+\mathrm{TT})$ in predicting MetS risk, using the lowest quartile of dietary pattern scores and the homozygote group with major allele as the reference category both in the whole population and in men and women separately. 
Table 1. Characteristics of the subjects with the metabolic syndrome (with MetS) and the controls (without MetS) of the Tehran Lipid and Glucose Study

(Mean values and standard deviations; $n$ 828)

\begin{tabular}{|c|c|c|c|c|}
\hline & \multicolumn{2}{|c|}{ With MetS } & \multicolumn{2}{|c|}{ Without MetS } \\
\hline & Mean & SD & Mean & SD \\
\hline Baseline age (years) & $42 \cdot 1$ & 12 & $42 \cdot 4$ & 13 \\
\hline Men $(n 470)$ & 40.7 & 13 & $41 \cdot 2$ & 13 \\
\hline Women (n 358) & 44.0 & 11 & $44 \cdot 0$ & 12 \\
\hline Current smokers (\%) & $12 \cdot 3$ & & $15 \cdot 0$ & \\
\hline Low physical activity (\%) & $36 \cdot 8$ & & 33.5 & \\
\hline Education level $\geq 14$ years (\%) & $13 \cdot 2$ & & $16 \cdot 3$ & \\
\hline Baseline BMI $\left(\mathrm{kg} / \mathrm{m}^{2}\right)$ & $26 \cdot 1^{*}$ & 4 & $22 \cdot 9$ & 4 \\
\hline Obesity (\%)† & $38 \cdot 6^{*}$ & & 3.6 & \\
\hline Baseline WC (cm) & $86 \cdot 2^{*}$ & 11 & $78 \cdot 0$ & 10 \\
\hline Abdominal obesity (\%) $\dagger$ & $74 \cdot 6^{\star}$ & & $5 \cdot 1$ & \\
\hline Baseline systolic BP (mmHg) & $114^{*}$ & 12 & 109 & 11 \\
\hline Baseline diastolic BP (mmHg) & $75 \cdot 0^{*}$ & 8 & 71.5 & 8 \\
\hline Elevated BP $(\%) \dagger$ & $43 \cdot 8^{\star}$ & & $2 \cdot 2$ & \\
\hline Baseline HDL-C (mmol/l) & $1.02^{*}$ & 0.26 & 1.23 & 0.26 \\
\hline Low HDL-C (\%)† & $78 \cdot 5^{\star}$ & & 1.4 & \\
\hline Baseline TAG $(\mathrm{mmol} / \mathrm{l})$ & $1.67^{*}$ & 0.84 & $1 \cdot 14$ & 0.50 \\
\hline High TAG $(\%) \dagger$ & $72 \cdot 0^{\star}$ & & 0.5 & \\
\hline Baseline FBG (mmol/l) & $5 \cdot 00^{*}$ & 0.72 & 4.81 & 0.56 \\
\hline High FBG $(\%) \dagger$ & $9 \cdot 4^{*}$ & & $1 \cdot 0$ & \\
\hline Energy intake $(\mathrm{kJ} / \mathrm{d})$ & 9765 & 4477 & 9795 & 4096 \\
\hline Carbohydrate ( $\%$ of energy) & 58.9 & 7 & $58 \cdot 8$ & 6 \\
\hline Total fat (\% of energy) & 29.9 & 6 & $30 \cdot 1$ & 6 \\
\hline SFA (\% of energy) & $9 \cdot 8$ & 3 & $10 \cdot 6$ & 10 \\
\hline MUFA (\% of energy) & $10 \cdot 2$ & 3 & $10 \cdot 1$ & 2 \\
\hline Fibre intake ( $g / 4184 \mathrm{~kJ}$ per $\mathrm{d})$ & $17 \cdot 9$ & 7 & $18 \cdot 1$ & 7 \\
\hline
\end{tabular}

WC, waist circumference; BP, blood pressure; HDL-C, HDL-cholesterol; FBG, fasting blood glucose.

* Mean value was significantly different from that of the control group $(P<0.05)$.

$\dagger \mathrm{BMI} \geq 30 \mathrm{~kg} / \mathrm{m}^{2}, W C \geq 95 \mathrm{~cm}$ for both sexes, elevated $B P \geq 130 / 85 \mathrm{mmHg}$, $\mathrm{HDL}-\mathrm{C}<1.04 \mathrm{mmol} / \mathrm{l}$ in men and $<1.30 \mathrm{mmol} / \mathrm{l}$ in women, $\mathrm{TAG} \geq 1.70 \mathrm{mmo} / \mathrm{l}$, and high $\mathrm{FBG} \geq 6.11 \mathrm{mmol} / \mathrm{l}$.

For diplotype combined analyses, rs670 (GA+AA) and rs5069 (CT + TT) were included in the analysis, compared with other genotype combinations (GG + CC). When APOC3 rs5128 was included in the analysis, the genotype combination comprised rs670 (GA + AA), rs5069 (CT + TT) and rs5128 (CC), compared with other combinations. Conditional logistic regression analysis was used to examine the combined role of quartiles of dietary pattern scores and genotype combinations in predicting MetS risk, which were adjusted for baseline BMI. To determine the $P$ value for trend across the quartiles of dietary pattern scores, logistic regression analysis was used with the median of each quartile of dietary pattern scores as a continuous variable.

\section{Results}

The mean age at baseline was $40 \cdot 7$ (SD13) and $41 \cdot 2$ (SD13) years in male cases and controls and $44 \cdot 0$ (SD11) and $44 \cdot 0$ (SD12) years in female cases and controls, respectively. The cases had a higher mean BMI than the controls (26.1 (SD4) v. 22.9 $\left.(\mathrm{sD} 4) \mathrm{kg} / \mathrm{m}^{2} ; P<0.05\right)$ at baseline. In subjects with the MetS, the highest prevalence of MetS risk factors was low HDL-C concentration (78.5\%) and abdominal obesity (74.6\%) (Table 1).

Genotype frequency did not deviate from Hardy-Weinberg equilibrium expectations, except for rs670 (Table 2).
Factor loadings of the three major dietary patterns are presented in Table 3.

Individuals with higher HDP scores were older and consumed less fat, sweets and sugar, and MUFA than those with lower HDP scores. A higher intake of fat, sweets and sugar was associated with higher WDP scores. The FSDP score was positively associated with the intake of total fat, SFA and MUFA, and inversely associated with the PUFA:SFA ratio (Table 4).

Adjusted OR for MetS risk according to quartile classification of dietary pattern scores by the dominant model of APOA1/ $A P O C 3$ genotypes are presented in Table 5. The risk of the MetS was not homogeneous in the two APOA1 genotypes across the quartiles of FSDP scores; the A allele carriers of rs670 and the $\mathrm{T}$ allele carriers of rs5069 had a lower odds of the MetS in the higher quartiles of FSDP scores compared with GG and CC genotypes of $\operatorname{rs670~}\left(P_{\text {interaction }}=0.01\right)$ and $\mathrm{rs} 5069$ $\left(P_{\text {interaction }}=0.05\right)$, respectively. After conducting this analysis separately by sex, the A allele carriers of rs670 had a lower odds of the MetS in the higher quartiles of FSDP scores compared with GG genotypes (men: GG genotype ( $n$ 204), OR 1, 7·36, $1 \cdot 60,2 \cdot 94, P_{\text {trend }}=0.17$ and GA + AA genotype $(n$ 266), OR 6.82, 5.95, 4.42, 2.26, $P_{\text {trend }}=0.02, P_{\text {interaction }}=0.01$; women: GG genotype ( $n$ 179), OR $1,0.87,0.50,1 \cdot 84, P_{\text {trend }}=0.71$ and $\mathrm{GA}+\mathrm{AA}$ genotype $(n$ 179), OR $1.47,1.53,0.73,0.35$, $\left.P_{\text {trend }}=0.02, P_{\text {interaction }}=0.06\right)$. In women, the T allele carriers of rs5069 had a lower odds of the MetS in the higher quartiles of FSDP scores compared with CC genotypes (CC genotype ( $n$ 235), OR 1 1 1 16, 0.54, 0.98, $P_{\text {trend }}=0.74$ and CT + TT genotype ( $n$ 123), OR 2.29, 1.36, 1.08, 0.53, $P_{\text {trend }}=0.05, P_{\text {interaction }}=0.34$; data not shown).

The examination of the individual WDP scores did not identify an interaction between dietary patterns and genetic

Table 2. Genotype and allele frequency of $A P O A 1$ and $A P O C 3$ polymorphisms in subjects with the metabolic syndrome (with MetS) and controls (without MetS) of the Tehran Lipid and Glucose Study (Number of alleles and genotype frequencies and percentages; $n$ 828)

\begin{tabular}{|c|c|c|c|c|}
\hline & \multicolumn{2}{|c|}{ With MetS } & \multicolumn{2}{|c|}{ Without MetS } \\
\hline & $n$ & $\%$ & $n$ & $\%$ \\
\hline \multicolumn{5}{|c|}{ Allele frequency rs 670} \\
\hline G & 589 & 71 & 604 & 73 \\
\hline A & 239 & 29 & 227 & 27 \\
\hline \multicolumn{5}{|c|}{ Genotype frequency rs670 } \\
\hline$G G$ & 186 & 45 & 197 & 47 \\
\hline GA & 217 & 52 & 210 & 51 \\
\hline AA & 11 & 3 & 7 & 2 \\
\hline \multicolumn{5}{|c|}{ Allele frequency rs5069 } \\
\hline C & 648 & 78 & 654 & 79 \\
\hline $\mathrm{T}$ & 180 & 22 & 174 & 21 \\
\hline \multicolumn{5}{|c|}{ Genotype frequency rs5069 } \\
\hline $\mathrm{CC}$ & 258 & 62 & 259 & 62 \\
\hline CT & 132 & 32 & 136 & 33 \\
\hline TT & 24 & 6 & 19 & 5 \\
\hline \multicolumn{5}{|c|}{ Allele frequency rs 5128} \\
\hline C & 645 & 81 & 655 & 81 \\
\hline G & 155 & 19 & 157 & 19 \\
\hline \multicolumn{5}{|c|}{ Genotype frequency rs5128 } \\
\hline $\mathrm{CC}$ & 261 & 65 & 261 & 44 \\
\hline CG & 123 & 31 & 133 & 33 \\
\hline GG & 16 & 4 & 12 & 3 \\
\hline
\end{tabular}


Table 3. Factor loadings for the three identified dietary patterns in the study participants of the Tehran Lipid and Glucose Study

\begin{tabular}{|c|c|c|c|}
\hline \multirow[b]{2}{*}{ Food groups } & \multicolumn{3}{|c|}{ Dietary patterns ${ }^{\star} \dagger$} \\
\hline & Healthy & Western & Fat-sweet \\
\hline Other vegetables & 0.77 & & \\
\hline Yellow-red vegetables & 0.74 & & \\
\hline Green vegetables & 0.60 & & 0.30 \\
\hline Fruits & 0.54 & & 0.22 \\
\hline Starchy vegetables & 0.50 & & \\
\hline Legumes & 0.47 & & -0.24 \\
\hline Low-fat dairy products & 0.41 & & \\
\hline Whole grains & 0.26 & & -0.32 \\
\hline Eggs & 0.21 & & 0.28 \\
\hline Liquid oils & 0.44 & 0.28 & \\
\hline Nuts and seeds & 0.42 & 0.27 & \\
\hline Fruit juice & 0.33 & 0.40 & \\
\hline Fish & 0.30 & 0.40 & \\
\hline Salty snacks & 0.25 & 0.58 & \\
\hline Poultry & 0.24 & 0.25 & \\
\hline Soft drinks & & 0.69 & \\
\hline Fast foods & & 0.66 & \\
\hline Sweets & & 0.49 & 0.31 \\
\hline Organ meat & & 0.44 & \\
\hline Red meat & & 0.23 & 0.42 \\
\hline Refined grains & & 0.21 & 0.27 \\
\hline Sugar & & & 0.64 \\
\hline Tea and coffee & & & 0.58 \\
\hline High-fat dairy products & & & 0.39 \\
\hline Solid oils & & & 0.38 \\
\hline Variance (\%) & $13 \cdot 2$ & $10 \cdot 2$ & $7 \cdot 7$ \\
\hline
\end{tabular}

variants of $A P O A 1$ and $A P O C 3$ genotypes. MetS risk appeared to increase significantly in the higher quartiles of WDP scores in the CC homozygote of $A P O C 3\left(P_{\text {trend }}=0.04\right)$ and in the A $\left(P_{\text {trend }}=0.008\right)$ and $\mathrm{T}\left(P_{\text {trend }}=0.03\right)$ allele carriers of $A P O A 1$ rs670 and rs5069, respectively. Also, the conditional logistic regression analysis conducted separately in men and women showed that this trend remains significant in men for the $A P O A 1 \mathrm{SNP}$. MetS risk increased in the higher quartiles of WDP scores and in the A allele carriers of $A P O A 1 \mathrm{rs} 670$ (GG genotype $\left(n\right.$ 204), OR 1, 2.24, 1.98, 1.11, $P_{\text {trend }}=0.22$ and GA + AA genotype ( $n$ 266), OR 1.44, 1.35, 2.62, 4.57, $\left.P_{\text {trend }}=0.001, \quad P_{\text {interaction }}=0.08\right)$ and the $\mathrm{T}$ allele carriers of $A P O A 1$ rs5069 (CC genotype ( $n$ 282), OR 1, 1.50, 1.04, $1 \cdot 22, P_{\text {trend }}=0.13$ and CT + TT genotype $(n$ 188), OR 0.37 , $\left.0.44,1.13,1.71, P_{\text {trend }}<0.001, P_{\text {interaction }}=0.11\right)$. MetS risk increased in the higher quartiles of WDP scores in all the genotypes of APOC3 rs5128 in men (CC genotype ( $n$ 304), OR 1 , $1.98,2.53,2.32, \quad P_{\text {trend }}=0.009$ and $\mathrm{CG}+\mathrm{GG}$ genotype ( $n$ 154), OR $1.40, \quad 0.85, \quad 1.71, \quad 3 \cdot 15, \quad P_{\text {trend }}=0.02$, $P_{\text {interaction }}=0.56$; data not shown).

Adherence to the HDP was associated with MetS risk in GG homozygote individuals of $\mathrm{rs670}\left(P_{\text {trend }}=0.008\right)$, an association that did not, however, remain significant after conducting this analysis separately in men and women. MetS risk decreased in the higher quartiles of HDP scores in the CC genotype of APOA1 rs5069 in women (CC genotype $\left(n\right.$ 235), OR $1,1.21,0.78,0.67, P_{\text {trend }}=0.50$ and CT + TT genotype ( $n$ 123), OR $0.29,0.66,1.36,1.96, P_{\text {trend }}=0.11$, $P_{\text {interaction }}=0.03$; data not shown).

HDP scores did not modulate the association of the combined genotype carriers of $A P O A 1$ and $A P O A 1 / A P O C 3$ (Table 6). The diplotype combination genotype of the APOA1 SNP (rs670/rs5069) interacted with adherence to WDP scores in determining the risk of developing the MetS $\left(P_{\text {interaction }}=0.03\right)$. The genotype carriers $(\mathrm{GA}+\mathrm{AA} /$ $\mathrm{CT}+\mathrm{TT})$ in the highest quartile of WDP scores had a greater risk of the MetS (OR 3.22, 95\% CI 1.21, 8.58) than those in the first quartile. The combined effect of the major homozygote genotype of $A P O C 3$ and the minor allele carriers of $A P O A 1$ showed a further increase in the risk of the MetS in the higher quartiles of WDP scores (OR 1, 2.49, 8.73, 6.32, $\left.P_{\text {trend }}<0.001\right)$ compared with the other combinations $\left(P_{\text {interaction }}=0.003\right)$. A significant interaction was found between the quartiles of FSDP scores and the APOA1 diplotype combination genotype (GA $+\mathrm{AA} / \mathrm{CT}+\mathrm{TT})$. OR for the genotype carriers were $1,0.65,0.57$ and $0.22\left(P_{\text {trend }}=0.006\right)$ in the highest to lowest quartiles of FSDP scores, compared with the other combined genotypes $\left(P_{\text {interaction }}=0.03\right)$. In the genotype combination of $\mathrm{GA}+\mathrm{AA} / \mathrm{CT}+\mathrm{TT} / \mathrm{CC}$, MetS risk was decreased in the higher quartiles of FSDP scores compared with the lower quartiles $\left(P_{\text {trend }}=0.03\right)$.

Analyses were carried out separately in men and women. In men, MetS risk was increased in the higher quartiles of WDP scores and the diplotype combination genotype of the APOA1 SNP (rs670/rs5069) (GA + AA/CT + TT ( $n$ 143), OR 1 , $1 \cdot 29,4 \cdot 48,5 \cdot 37, P_{\text {trend }}=0.001$ and other genotypes $(n 327)$, OR $2 \cdot 61,4 \cdot 18,3 \cdot 14,3 \cdot 61, P_{\text {trend }}=0.06, P$ interaction=0.06). In women, the genotype carriers $\mathrm{GA}+\mathrm{AA} / \mathrm{CT}+\mathrm{TT}$ in the highest quartile of FSDP scores had a lower risk of the MetS $\left(\mathrm{GA}+\mathrm{AA} / \mathrm{CT}+\mathrm{TT}(n 86), \mathrm{OR} 1,0 \cdot 89,0 \cdot 55,0 \cdot 08, P_{\text {trend }}=0.03\right.$ and other genotypes ( $n$ 272), OR $0.59,0.55,0.28,0.61$, $P_{\text {trend }}=0 \cdot 77, P$ interaction $=0 \cdot 04$; data not shown).

\section{Discussion}

In the present nested case-control study of Tehranian adults, we assessed the interactions between dietary patterns and genetic variants of $A P O A 1 / A P O C 3$ SNP (rs670, rs5069/ rs5128) in relation to MetS risk. The WDP interacted with combined $A P O A 1 / A P O C 3$ genotypes in relation to the risk of the MetS. Intakes of the WDP were associated with a higher risk of the MetS among individuals having the major homozygote genotype of $A P O C 3$ and the minor allele carriers of $A P O A 1$. When sexes were analysed separately, differences in MetS risk became more obvious in men than in women. A significant interaction was also found between the FSDP and the APOA1 diplotypic variable in relation to the risk of the MetS. Decreased MetS risk was observed in individuals with both minor allele carriers of the APOA1 SNP and higher FSDP scores. When sexes were analysed separately, differences in MetS risk became more obvious in women than in men.

Participants with a higher adherence to the HDP had a high risk of the MetS if they were the carriers of the GG genotype or rs670. This may be due to the subjects with the GG genotype being more susceptible to higher energy and cholesterol 
Table 4. Dietary intakes of the participants by quartiles $(Q)$ of dietary pattern scores*

(Mean values with their standard errors)

\begin{tabular}{|c|c|c|c|c|c|c|c|c|c|}
\hline \multirow[b]{2}{*}{ Dietary intakes } & \multicolumn{2}{|c|}{ Q1 } & \multicolumn{2}{|c|}{ Q2 } & \multicolumn{2}{|c|}{ Q3 } & \multicolumn{2}{|c|}{ Q4 } & \multirow[b]{2}{*}{$P$} \\
\hline & Mean & SEM & Mean & SEM & Mean & SEM & Mean & SEM & \\
\hline \multicolumn{10}{|l|}{ Fat (\% of energy) } \\
\hline Healthy DP & $29 \cdot 4$ & 0.4 & $31 \cdot 3$ & 0.4 & $29 \cdot 8$ & 0.4 & $29 \cdot 7$ & 0.4 & $<0.01$ \\
\hline Western DP & $28 \cdot 3$ & 0.4 & $29 \cdot 6$ & 0.4 & 31.4 & 0.4 & $31 \cdot 0$ & 0.4 & $<0.001$ \\
\hline Fat-sweet DP & $28 \cdot 6$ & 0.4 & $30 \cdot 0$ & 0.4 & 30.5 & 0.4 & $31 \cdot 1$ & 0.4 & $<0.001$ \\
\hline \multicolumn{10}{|l|}{ SFA (\% of energy) } \\
\hline Healthy DP & $10 \cdot 9$ & 0.5 & $10 \cdot 2$ & 0.5 & $8 \cdot 3$ & 0.5 & 9.8 & 0.5 & 0.32 \\
\hline Western DP & $10 \cdot 0$ & 0.5 & $10 \cdot 9$ & 0.5 & $10 \cdot 3$ & 0.5 & $9 \cdot 7$ & 0.5 & 0.35 \\
\hline Fat-sweet DP & $9 \cdot 1$ & 0.5 & $10 \cdot 3$ & 0.5 & $10 \cdot 0$ & 0.5 & 11.5 & 0.5 & $<0.05$ \\
\hline \multicolumn{10}{|l|}{ Fibre $(\mathrm{g} / \mathrm{d})$} \\
\hline Healthy DP & 14.9 & 0.4 & $16 \cdot 2$ & 0.4 & $17 \cdot 6$ & 0.4 & $19 \cdot 5$ & 0.4 & $<0.001$ \\
\hline Western DP & $18 \cdot 2$ & 0.5 & $17 \cdot 7$ & 0.4 & $16 \cdot 6$ & 0.4 & $15 \cdot 9$ & 0.5 & $<0.01$ \\
\hline Fat-sweet DP & $19 \cdot 5$ & 0.4 & $17 \cdot 5$ & 0.4 & $16 \cdot 4$ & 0.4 & 14.9 & 0.4 & $<0.001$ \\
\hline \multicolumn{10}{|c|}{ Sweets and sugar $(g / d)$} \\
\hline Healthy DP & $45 \cdot 8$ & 3 & 38.9 & 2 & 38.9 & 2 & $33 \cdot 8$ & 2 & $<0.01$ \\
\hline Western DP & $30 \cdot 5$ & 3 & 35.4 & 2 & 42.9 & 2 & $48 \cdot 6$ & 3 & $<0.001$ \\
\hline Fat-sweet DP & $18 \cdot 4$ & 2 & 29.5 & 2 & 43.6 & 2 & $67 \cdot 3$ & 2 & $<0.001$ \\
\hline \multicolumn{10}{|c|}{ MUFA ( $\%$ of energy) } \\
\hline Healthy DP & $10 \cdot 1$ & 0.2 & $10 \cdot 6$ & 0.2 & $10 \cdot 1$ & 0.2 & $9 \cdot 8$ & 0.2 & $<0.01$ \\
\hline Western DP & $9 \cdot 8$ & 0.2 & $10 \cdot 0$ & 0.2 & $10 \cdot 7$ & 0.2 & $10 \cdot 4$ & 0.2 & $<0.01$ \\
\hline Fat-sweet DP & 9.5 & 0.2 & $10 \cdot 2$ & 0.2 & 10.5 & 0.2 & $10 \cdot 6$ & 0.2 & $<0.001$ \\
\hline \multicolumn{10}{|l|}{ PUFA:SFA ratio } \\
\hline Healthy DP & 0.59 & 0.03 & 0.64 & 0.02 & 0.60 & 0.02 & 0.59 & 0.02 & $0 \cdot 14$ \\
\hline Western DP & 0.59 & 0.02 & 0.60 & 0.02 & 0.63 & 0.02 & 0.63 & 0.02 & 0.54 \\
\hline Fat-sweet DP & 0.66 & 0.02 & 0.59 & 0.02 & 0.63 & 0.02 & 0.58 & 0.02 & $<0.01$ \\
\hline \multicolumn{10}{|l|}{$\mathrm{CHO}$ (\% of energy) } \\
\hline Healthy DP & $58 \cdot 7$ & & $57 \cdot 2$ & & $59 \cdot 2$ & & $58 \cdot 7$ & & 0.03 \\
\hline Western DP & $60 \cdot 6$ & & $58 \cdot 6$ & & $56 \cdot 9$ & & $57 \cdot 2$ & & $<0.01$ \\
\hline Fat-sweet DP & $59 \cdot 6$ & & $58 \cdot 1$ & & $57 \cdot 7$ & & 57.9 & & 0.03 \\
\hline \multicolumn{10}{|c|}{ Vegetable SFA (\% of energy) } \\
\hline Healthy DP & 2.92 & 0.4 & $2 \cdot 25$ & 0.3 & 1.96 & 0.3 & $1 \cdot 16$ & 0.3 & $<0.01$ \\
\hline Western DP & $2 \cdot 12$ & 0.4 & 2.47 & 0.3 & 1.82 & 0.4 & 1.77 & 0.4 & 0.27 \\
\hline Fat-sweet DP & 0.60 & 0.3 & 1.70 & 0.3 & 2.35 & 0.3 & $3 \cdot 36$ & 0.3 & $<0.01$ \\
\hline \multicolumn{10}{|l|}{ Cholesterol (g/d) } \\
\hline Healthy DP & 240 & 15 & 249 & 14 & 261 & 14 & 293 & 15 & $<0.01$ \\
\hline Western DP & 222 & 14 & 243 & 14 & 266 & 14 & 319 & 14 & $<0.01$ \\
\hline Fat-sweet DP & 254 & 14 & 250 & 14 & 257 & 14 & 274 & 14 & 0.47 \\
\hline \multicolumn{10}{|l|}{ Energy $(\mathrm{kJ} / \mathrm{d})$} \\
\hline Healthy DP & 8698 & 427 & 9485 & 389 & 9937 & 402 & 9895 & 418 & 0.02 \\
\hline Western DP & 9590 & 418 & 9694 & 397 & 9845 & 402 & 8958 & 423 & 0.22 \\
\hline Fat-sweet DP & 10079 & 406 & 9364 & 410 & 8795 & 402 & 9970 & 406 & 0.01 \\
\hline
\end{tabular}

intakes. In the present study, adherence to the HDP was positively associated with energy intake. Thus, the beneficial effects of the HDP in the prevention of the MetS might be seen more in A allele carriers than in GG genotype subjects. Previous studies have shown that the relationship between energy intake and obesity differed among subjects with different genotypes of the IL-6 receptor (IL6R) gene Asp358Ala $(\mathrm{T} / \mathrm{G})$ polymorphism in Japanese men ${ }^{(24)}$ and the Trp64Arg polymorphism of the $\beta 3$-adrenergic receptor (ADRB3) gene ${ }^{(25)}$. This observation can be explained by the fact that total energy intake is more relevant than dietary fat intake, and people merely replace energy from fat with energy from carbohydrates ${ }^{(26)}$. Although the mean cholesterol intakes in all the quartiles of HDP scores were in accordance with the recommended guidelines, higher intakes of cholesterol in the higher quartiles of HDP scores may increase the risk of the MetS in the GG genotype of rs670 subjects, an association which might be dependent on cholesterol intake.

In women with the major homozygote of APOA1 rs5069, higher quartiles of HDP scores were associated with a lower risk of the MetS, compared with minor allele carriers who had an increased risk of the MetS.

In all the participants, higher intakes of the WDP were found to be related to an increased risk of the MetS in A and T allele carriers of the APOA1 polymorphism and the CC homozygote of the $A P O C 3$ polymorphism. In addition, these analyses were stratified by sex, and in men, the increasing trends of MetS risk remained significant. The present results on the WDP and the MetS are consistent with those of a previous study, in which intakes of the WDP were associated with the risk of developing diabetes among men with a higher genotype risk score ${ }^{(27)}$, a finding that highlights the high variability of MetS risk in individuals with different combined 
Table 5. Adjusted OR for the metabolic syndrome according to quartile (Q) classification of dietary pattern (DP) scores by the dominant model of APOA1/APOC3 genotypes*

(Odds ratios and $95 \%$ confidence intervals)

\begin{tabular}{|c|c|c|c|c|c|c|c|c|c|c|}
\hline & \multicolumn{2}{|c|}{ Q1 } & \multicolumn{2}{|r|}{ Q2 } & \multicolumn{2}{|r|}{ Q3 } & \multicolumn{2}{|c|}{ Q4 } & \multirow[b]{2}{*}{$P_{\text {trend }}$} & \multirow[b]{2}{*}{$P_{\text {interaction }}$} \\
\hline & OR & $95 \% \mathrm{Cl}$ & OR & $95 \% \mathrm{Cl}$ & OR & $95 \% \mathrm{Cl}$ & OR & $95 \% \mathrm{Cl}$ & & \\
\hline \multicolumn{11}{|l|}{ APOA1 genotypes (rs670) } \\
\hline Healthy DP & & & & & & & & & & 0.15 \\
\hline $\mathrm{GG}(n 383)$ & 1 (Ref) & & 1.35 & $0.64,2 \cdot 84$ & 1.45 & $0.72,2.93$ & 1.76 & $0.87,3.56$ & 0.008 & \\
\hline $\mathrm{GA}+\mathrm{AA}(n 445)$ & $2 \cdot 35$ & $1 \cdot 12,4.90$ & $1 \cdot 13$ & $0.55,2.31$ & 1.87 & $0.93,3.72$ & 1.53 & $0.75,3.08$ & 0.60 & \\
\hline Western DP & & & & & & & & & & 0.06 \\
\hline $\mathrm{GG}(n 383)$ & 1 & & 0.94 & $0.43,2.07$ & 0.61 & $0.29,1.27$ & 0.85 & $0.40,1.83$ & 0.42 & \\
\hline $\mathrm{GA}+\mathrm{AA}(n 445)$ & 0.65 & $0.32,1.31$ & 0.85 & $0.43,1.66$ & 1.24 & $0.60,2.56$ & 1.66 & $0.80,3.47$ & 0.008 & \\
\hline Fat-sweet DP & & & & & & & & & & 0.01 \\
\hline $\mathrm{GG}(n$ 383) & 1 & & 1.90 & $0.95,3.79$ & 0.86 & $0.42,1.73$ & 1.74 & $0.79,3.84$ & 0.27 & \\
\hline $\mathrm{GA}+\mathrm{AA}(n 445)$ & 2.42 & $1 \cdot 17,5 \cdot 00$ & 2.46 & $1.22,4.92$ & 1.44 & $0.72,2.90$ & 0.81 & $0.39,1.70$ & $<0.01$ & \\
\hline \multicolumn{11}{|l|}{ APOA1 genotypes (rs5069) } \\
\hline Healthy DP & & & & & & & & & & $0 \cdot 17$ \\
\hline $\mathrm{CC}(n 517)$ & 1 & & 0.87 & $0.47,1.62$ & 0.89 & $0.47,1.66$ & 0.83 & $0.44,1.52$ & 0.48 & \\
\hline $\mathrm{CT}+\mathrm{TT}(n$ 311) & 0.64 & $0.31,1.36$ & 0.48 & $0.23,0.96$ & 1.04 & $0.54,2.02$ & 1.22 & $0.60,2.47$ & 0.11 & \\
\hline Western DP & & & & & & & & & & 0.35 \\
\hline CC $(n 517)$ & 1 & & 1.04 & $0.58,1.89$ & 0.80 & $0.42,1.52$ & $1 \cdot 13$ & $0.59,2.18$ & 0.93 & \\
\hline $\mathrm{CT}+\mathrm{TT}(n$ 311) & 0.57 & $0.28,1.14$ & $0 \cdot 81$ & $0.40,1.62$ & 1.01 & $0.49,2.08$ & 1.40 & $0.67,2.93$ & 0.03 & \\
\hline Fat-sweet DP & & & & & & & & & & 0.05 \\
\hline CC $(n 517)$ & 1 & & 1.98 & $1.07,3.65$ & 0.85 & $0.46,1.60$ & $1 \cdot 18$ & $0.61,2 \cdot 28$ & 0.92 & \\
\hline $\mathrm{CT}+\mathrm{TT}(n 311)$ & 1.85 & $0.88,3.88$ & $1 \cdot 34$ & $0.65,2.73$ & 1.08 & $0.53,2 \cdot 20$ & 0.55 & $0.26,1.16$ & 0.005 & \\
\hline \multicolumn{11}{|l|}{ APOC3 genotype (rs5128) } \\
\hline Healthy DP & & & & & & & & & & 0.18 \\
\hline CC (n 522) & 1 & & 1.06 & $0.57,1.98$ & $1 \cdot 10$ & $0.60,2.00$ & 1.07 & $0.57,2.02$ & 0.25 & \\
\hline $\mathrm{CG}+\mathrm{GG}(n 284)$ & $1 \cdot 28$ & $0.58,2.80$ & 0.45 & $0.21,0.94$ & $1 \cdot 12$ & $0.53,2.33$ & 0.98 & $0.49,1.97$ & 0.37 & \\
\hline Western DP & & & & & & & & & & $0 \cdot 12$ \\
\hline CC (n 522) & 1 & & 1.61 & $0.87,3.02$ & 1.57 & $0.80,3.05$ & 1.98 & $1.01,3.90$ & 0.04 & \\
\hline $\mathrm{CG}+\mathrm{GG}(n$ 284) & 1.65 & $0.76,3.60$ & 0.71 & $0.32,1.56$ & 1.22 & $0.59,2.54$ & 1.89 & $0.81,4.40$ & 0.93 & \\
\hline Fat-sweet DP & & & & & & & & & & 0.70 \\
\hline CC (n 522) & 1 & & $1 \cdot 12$ & $0.61,2.05$ & 0.58 & $0.30,1 \cdot 13$ & 0.62 & $0.33,1.19$ & 0.09 & \\
\hline$C G+G G(n 284)$ & 0.65 & $0.32,1.35$ & 1.03 & $0.48,2.21$ & 0.66 & $0.33,1.32$ & 0.43 & $0.18,1.03$ & 0.41 & \\
\hline
\end{tabular}

APOA1, rs670 (-75G > A), rs5069 (+83C > T); Ref, reference; APOC3, rs5128 $(3238 \mathrm{C}>\mathrm{G})$.

*OR were calculated using the conditional logistic regression model, adjusted for baseline BMI and the interaction term (SNP $\times$ DP scores). Participants were jointly classified (eight groups) according to quartiles of DP scores and dominant model of the rs5128, rs670 and rs5069 genotypes. The lowest quartile of DP scores and homozygote genotype with major allele were used as the reference group.

genotypes and may be due to different lifestyles. The effect of the WDP on the risk of the MetS is not homogeneous in individuals with different genetic backgrounds. In the present study, individuals consuming a higher WDP had higher intakes of total fat, MUFA and cholesterol. Consistent with a previous study in this population, fast food consumption was associated with the risk of the MetS, which might be due to poor diet quality, higher energy density and glycaemic index, high $\mathrm{Na}$, trans-fatty acid and refined carbohydrate intakes, and the decrease in dietary phytochemical index and dietary total antioxidant capacity, being the main dietary risk factors of the $\operatorname{MetS}^{(28)}$. In addition, processing of these foods generate toxic chemicals that induce insulin resistance $^{(29,30)}$. However, the molecular mechanisms underlying these gene-nutrient interactions remain unclear, and functional studies are needed to ascertain biological significance. Previous studies have shown that the genetic association between APOA1 rs670 and MetS risk was modulated by dietary fat intake and composition. These studies have also reported that A allele carriers of $A P O A 1$ rs670 were more responsive and susceptible to dietary fat change than the GG homozygote ${ }^{(31,32)}$. Studies have reported vague results regarding the effects of these polymorphisms on MetS risk in the context of different dietary patterns. The diplotypic combined effect of genotypes exacerbated the interaction with the WDP in relation to MetS risk; however, when A and T alleles were taken into account, the genotype combination $\mathrm{GA}+\mathrm{AA} / \mathrm{CT}+\mathrm{TT}$ exhibited higher MetS risk with a higher WDP score. The deleterious effects conferred by the diplotype combination of the APOA1 polymorphism were aggregated among individuals with higher scores of the WDP and $\mathrm{GA}+\mathrm{AA} / \mathrm{CT}+\mathrm{TT} / \mathrm{CC}$ genotype combination of APOA1 rs670/rs5069/APOC3 rs5128, suggesting that these individuals were more susceptible to the effects of the WDP than others.

Intakes of the FSDP, which were characterised by a high consumption of tea and coffee, sugar and sweets, red meat, high-fat dairy products, and solid oils, showed a significant interaction with $A P O A 1$ rs670, rs5069 and their diplotype combination, in relation to MetS risk. The OR for MetS risk was decreased in $\mathrm{A}$ and $\mathrm{T}$ allele carriers with an increase in FSDP scores; however, when sexes were analysed separately, differences in MetS risk became more obvious in women than in men. Previous studies focusing on the modulation of a specific dietary fat in relation to the association between polymorphisms and MetS risk or blood lipids found that distinguishing these relationships is difficult because of collinearity of different kinds of fat intakes. Dietary pattern analysis examines the multiple dimensions of foods simultaneously 
Table 6. Adjusted OR for the metabolic syndrome and combined $A P O A 1 / A P O C 3$ genotypes according to quartile (Q) classification of dietary pattern (DP) scores*

(Odds ratios and $95 \%$ confidence intervals)

\begin{tabular}{|c|c|c|c|c|c|c|c|c|c|c|}
\hline & \multicolumn{2}{|r|}{ Q1 } & \multicolumn{2}{|r|}{ Q2 } & \multicolumn{2}{|r|}{ Q3 } & \multicolumn{2}{|r|}{ Q4 } & \multirow[b]{2}{*}{$P_{\text {trend }}$} & \multirow[b]{2}{*}{$P_{\text {interaction }}$} \\
\hline & OR & $95 \% \mathrm{Cl}$ & OR & $95 \% \mathrm{Cl}$ & OR & $95 \% \mathrm{Cl}$ & OR & $95 \% \mathrm{Cl}$ & & \\
\hline Healthy DP & & & & & & & & & & 0.19 \\
\hline $\mathrm{GA}+\mathrm{AA} / \mathrm{CT}+\mathrm{TT}(n$ 229) & 1 & & 0.65 & $0.25,1.66$ & 1.07 & $0.41,2.85$ & $2 \cdot 05$ & $0.74,5.67$ & 0.48 & \\
\hline Others $(n 599)$ & 1.33 & $0.58,3.09$ & $1 \cdot 13$ & $0.49,2.61$ & 1.49 & $0.65,3.40$ & 1.20 & $0.52,2.78$ & 0.20 & \\
\hline Western DP & & & & & & & & & & 0.03 \\
\hline $\mathrm{GA}+\mathrm{AA} / \mathrm{CT}+\mathrm{TT}(n 229)$ & 1 & & 1.59 & $0.63,4.05$ & 3.32 & $1.26,8.79$ & 3.22 & $1.21,8.58$ & 0.008 & \\
\hline Others ( $n$ 599) & $2 \cdot 35$ & $1.12,4.95$ & $2 \cdot 50$ & $1 \cdot 10,5 \cdot 66$ & 1.67 & $0.76,3.70$ & $2 \cdot 67$ & $1 \cdot 18,6 \cdot 05$ & 0.94 & \\
\hline Fat-sweet DP & & & & & & & & & & 0.03 \\
\hline $\mathrm{GA}+\mathrm{AA} / \mathrm{CT}+\mathrm{TT}(n$ 229) & 1 & & 0.65 & $0.25,1.68$ & 0.57 & $0.23,1.38$ & 0.22 & $0.08,0.60$ & 0.006 & \\
\hline Others ( $n$ 599) & 0.54 & $0.24,1 \cdot 21$ & 0.99 & $0.47,2.09$ & 0.43 & $0.19,0.98$ & 0.61 & $0.27,1.39$ & 0.71 & \\
\hline Healthy DP & & & & & & & & & & 0.73 \\
\hline $\mathrm{GA}+\mathrm{AA} / \mathrm{CT}+\mathrm{TT} / \mathrm{CC}(n 147)$ & 1 & & 0.97 & $0.31,3.06$ & 0.96 & $0.30,3.04$ & 1.44 & $0.42,5.00$ & 0.94 & \\
\hline Others ( $n$ 659) & $1 \cdot 19$ & $0.46,3 \cdot 10$ & 0.76 & $0.30,1.95$ & 1.28 & $0.49,3.30$ & 1.09 & $0.42,2.83$ & 0.1 & \\
\hline Western DP & & & & & & & & & & 0.003 \\
\hline $\mathrm{GA}+\mathrm{AA} / \mathrm{CT}+\mathrm{TT} / \mathrm{CC}(n 147)$ & 1 & & 2.49 & $0.78,7.96$ & 8.73 & $2 \cdot 33,32 \cdot 65$ & $6 \cdot 32$ & $1.82,21.93$ & $<0.001$ & \\
\hline Others $(n 659)$ & 3.01 & $1 \cdot 24,7 \cdot 27$ & $2 \cdot 69$ & $1.05,6.89$ & $2 \cdot 34$ & $0.93,6.88$ & 3.42 & $1.33,5.95$ & 0.91 & \\
\hline Fat-sweet DP & & & & & & & & & & 0.40 \\
\hline $\mathrm{GA}+\mathrm{AA} / \mathrm{CT}+\mathrm{TT} / \mathrm{CC}(n 147)$ & 1 & & 0.68 & $0.19,2.40$ & 0.51 & $0.15,1.70$ & 0.27 & $0.08,0.93$ & 0.03 & \\
\hline Others $(n 659)$ & 0.52 & $0.19,1.42$ & 0.77 & $0.29,2.03$ & 0.40 & $0.14,1.14$ & 0.43 & $0.15,1.20$ & 0.27 & \\
\hline
\end{tabular}

APOA1, rs670 (-75G > A), rs5069 (+83C > T); APOC3, rs5128 $(3238 \mathrm{C}>\mathrm{G})$.

* OR were calculated using the conditional logistic regression model, adjusted for baseline BMI and the interaction term (SNP $\times$ DP scores). Participants were jointly classified (eight groups) according to quartiles of DP scores and risk genotype carriers of rs670 (GA+AA) and rs5069 (CT+TT). The lowest quartile of DP scores and risk genotype carriers were used as the reference group. When $A P O C 3$ rs5128 was included in the analysis, the risk genotype combination comprised rs670 (GA+AA), rs5069 $(\mathrm{CT}+\mathrm{TT})$ and rs5128 (CC).

relative to a given outcome ${ }^{(33)}$. In the present study, individuals with the highest FSDP score had higher intakes of SFA and MUFA, lower PUFA:SFA ratio, and lower intakes of carbohydrate and energy; these combinations reduced the risk of the MetS in subjects carrying the A and $\mathrm{T}$ alleles. Previously, the Framingham offspring study showed a significant interaction between dietary PUFA intake and the rs670 polymorphism in relation to plasma HDL-C concentration. In women carrying the A allele, a higher PUFA intake $(>8 \%$ of energy) was associated with a higher concentration of HDL-C; however, a moderate consumption of PUFA, common in most populations, revealed no phenotypegenotype association $^{(7)}$. In another study, the total fat intake ( $>35 \%$ of energy) or high MUFA intake ( $>14 \%$ of energy) accentuated the risk conferred by the GG genotype, whereas dietary PUFA and SFA did not modulate the risk of the MetS $^{(9)}$. Based on the present results, it seems that $\mathrm{A}$ and $\mathrm{T}$ allele carriers of the $A P O A 1$ polymorphism may benefit from the protective effect of the intake of dietary fat composition that is approximately in the range of recommended dietary guidelines $^{(34)}$ (e.g. SFA, $10-11 \%$ of energy ( $4 \%$ from vegetable SFA); MUFA, 10-11\% of energy intake; cholesterol $<300 \mathrm{~g} / \mathrm{d}$ ), although intakes of sweets and sugar increased in the higher quartiles of FSDP scores, but intake of carbohydrate was in the range of 57-60\% and energy intake did not increase in the higher quartiles of FSDP scores.

The mechanism underlying the associations between these genetic variants and the MetS is unknown; however, this dietary pattern could interact differently with the transcriptional apparatus of the $A P O A 1$ gene, increasing more transcription in the presence of $\mathrm{A}$ and $\mathrm{T}$ allele carriers ${ }^{(35)}$. Sex differences observed in dietary pattern interactions and the MetS might be related to hormonal effects. Thyroid hormones, glucocorticoids and oestradiol enhance the activity of the APOA1 gene, whereas androgens decrease it. The mechanisms that mediate the effects of the hormones include the direct effects of the ligand and the nuclear receptor complex on gene activity. However, indirect means involving the participation of transcription factors other than the hormone receptors are also possible. The regulation of the $A P O A 1$ gene depends not only on the presence of oestrogen receptor- $\alpha$ and $17 \beta$-oestradiol, but also on the intracellular balance of oestrogen receptor- $\alpha$ and co-activators used by oestrogen receptor- $\alpha$ and the APOA1 enhancer. The mutation G-A rs670 polymorphism might be in the promoter or enhancer region of the $A P O A 1$ gene ${ }^{(7,36,37)}$.

The strengths of the present study include a longitudinal design, large number of cases and matched controls from all demographic strata, and the use of a dietary pattern analysis that better detects the association of the overall diet composition and genetic predisposition with the risk of the MetS. The results of the present study also emphasises the importance of taking into account the gene-diet interaction effect in association studies. Assessing dietary intakes at just one time point is one of the limitations of the present study; however, assessment performed in the present study showed dietary patterns 1 year preceding the development of the MetS. Excluding individuals who had any dietary change due to the presence of MetS components reduces the possibility of any change in dietary behaviour in recent years. Although factor analysis was used to define dietary patterns, it was somewhat subjective; however, our previous study showed reproducibility, validity and stability of the WDP in a subsample of a Tehranian population ${ }^{(38)}$. Insulin sensitivity was not measured; therefore, we did not detect any interactions between dietary patterns and genotypes in 
relation to it, as a sensitive marker of the MetS. Replication studies carried out in a variety of populations are needed to indicate that this association is not limited to specific populations.

In conclusion, our findings suggest that dietary patterns modulate the effect of $A P O A 1$ and $A P O C 3 \mathrm{SNP}$ in relation to the risk of the MetS, with A and T allele carriers of $A P O A 1$ (rs670, rs5069) and C allele carriers of $A P O C 3$ (rs5128) further increasing the risk of the MetS when individuals adhered to the WDP. Also, A and T alleles of APOA1 appear to be protective against the risk of the MetS in participants with higher scores of the FSDP, which supports current dietary guidelines.

\section{Acknowledgements}

The authors acknowledge Ms Nilufar Shiva for critical editing of English grammar and syntax of this manuscript. The present study was conducted as a PhD thesis (Grant No. 407) and supported by the Research Institute for Endocrine Sciences, SBMU, Tehran, Iran.

The authors' contributions are as follows: F. H.-E., M. S. D., M. S., Y. M. and M. H. designed the study, collected and analysed the data, and wrote the manuscript; F. A. and P. M. supervised the research and critically revised the manuscript for important intellectual content.

None of the authors has any conflict of interest to declare.

\section{References}

1. Teran-Garcia M \& Bouchard C (2007) Genetics of the metabolic syndrome. Appl Physiol Nutr Metab 32, 89-114.

2. Joy T, Lahiry P, Pollex RL, et al. (2008) Genetics of metabolic syndrome. Curr Diab Rep 8, 141-148.

3. Albahrani AI, Usher JJ, Alkindi M, et al. (2007) ApolipoproteinA1-75 G/A (M1-) polymorphism and lipoprotein(a); anti- vs. pro-Atherogenic properties. Lipids Health Dis 6, 19.

4. Chen ES, Mazzotti DR, Furuya TK, et al. (2009) Apolipoprotein A1 gene polymorphisms as risk factors for hypertension and obesity. Clin Exp Med 9, 319-325.

5. Dodani S, Henkhaus R, Wick J, et al. (2011) Metabolic syndrome in South Asian immigrants: more than low HDL requiring aggressive management. Lipids Health Dis 10, 45.

6. Olivieri O, Bassi A, Stranieri C, et al. (2003) Apolipoprotein C-III, metabolic syndrome, and risk of coronary artery disease. J Lipid Res 44, 2374-2381.

7. Ordovas JM, Corella D, Cupples LA, et al. (2002) Polyunsaturated fatty acids modulate the effects of the APOA1 G-A polymorphism on HDL-cholesterol concentrations in a sex-specific manner: the Framingham Study. Am J Clin Nutr 75, 38-46.

8. Phillips CM, Tierney AC \& Roche HM (2008) Gene-nutrient interactions in the metabolic syndrome. J Nutrigenet Nutrigenomics 1, 136-151.

9. Phillips CM, Goumidi L, Bertrais S, et al. (2011) Gene-nutrient interactions and gender may modulate the association between $A p O A 1$ and $A p O B$ gene polymorphisms and metabolic syndrome risk. Atherosclerosis 214, 408-414.

10. Phillips CM (2013) Nutrigenetics and metabolic disease: current status and implications for personalised nutrition. Nutrients 5, 32-57.
11. Martinez-Gonzalez MA \& Martin-Calvo N (2013) The major European dietary patterns and metabolic syndrome. Rev Endocr Metab Disord 14, 265-271.

12. Azizi F, Ghanbarian A, Momenan AA, et al. (2009) Prevention of non-communicable disease in a population in nutrition transition: Tehran Lipid and Glucose Study phase II. Trials 10, 5.

13. Esfahani FH, Asghari G, Mirmiran P, et al. (2010) Reproducibility and relative validity of food group intake in a food frequency questionnaire developed for the Tehran Lipid and Glucose Study. J Epidemiol 20, 150-158.

14. Mirmiran P, Esfahani FH, Mehrabi Y, et al. (2010) Reliability and relative validity of an FFQ for nutrients in the Tehran lipid and glucose study. Public Health Nutr 13, 654-662.

15. Hosseini-Esfahani F, Djazaieri SA, Mirmiran P, et al. (2012) Which food patterns are predictors of obesity in Tehranian adults? J Nutr Educ Behav 44, 564-573.

16. Ainsworth BE, Haskell WL, Whitt MC, et al. (2000) Compendium of physical activities: an update of activity codes and MET intensities. Med Sci Sports Exerc 32, S498-S504.

17. Kriska AM, Knowler WC, LaPorte RE, et al. (1990) Development of questionnaire to examine relationship of physical activity and diabetes in Pima Indians. Diabetes Care 13, 401-411.

18. Momenan AA, Delshad M, Sarbazi N, et al. (2012) Reliability and validity of the Modifiable Activity Questionnaire (MAQ) in an Iranian urban adult population. Arch Iran Med 15, 279-282.

19. Daneshpour MS, Faam B, Mansournia MA, et al. (2012) Haplotype analysis of $A p O A I-C I I I-A I V$ gene cluster and lipids level: Tehran Lipid and Glucose Study. Endocrine 41, 103-110.

20. Truett GE, Heeger P, Mynatt RL, et al. (2000) Preparation of PCR-quality mouse genomic DNA with hot sodium hydroxide and tris (HotSHOT). Biotechniques 29, 52-54.

21. Zou Y, Hu D, Yang X, et al. (2003) Relationships among apolipoprotein A1 gene polymorphisms, lipid levels and coronary atherosclerosis disease. Chin Med J (Engl) 116, 665-668.

22. Azizi F, Hadaegh F, Khalili D, et al. (2010) Appropriate definition of metabolic syndrome among Iranian adults: report of the Iranian National Committee of Obesity. Arch Iran Med 13, 426-428.

23. Grundy SM, Hansen B, Smith SC Jr, et al. (2004) Clinical management of metabolic syndrome: report of the American Heart Association/National Heart, Lung, and Blood Institute/ American Diabetes Association conference on scientific issues related to management. Arterioscler Thromb Vasc Biol 24, e19-e24.

24. Song Y, Miyaki K, Araki J, et al. (2007) The interaction between the interleukin 6 receptor gene genotype and dietary energy intake on abdominal obesity in Japanese men. Metabolism 56, 925-930.

25. Miyaki K, Sutani S, Kikuchi H, et al. (2005) Increased risk of obesity resulting from the interaction between high energy intake and the Trp64Arg polymorphism of the beta3-adrenergic receptor gene in healthy Japanese men. J Epidemiol 15, 203-210.

26. Sanchez-Moreno C, Ordovas JM, Smith CE, et al. (2011) $A P O A 5$ gene variation interacts with dietary fat intake to modulate obesity and circulating triglycerides in a Mediterranean population. $J$ Nutr 141, 380-385.

27. Qi L, Cornelis MC, Zhang C, et al. (2009) Genetic predisposition, Western dietary pattern, and the risk of type 2 diabetes in men. Am J Clin Nutr 89, 1453-1458.

28. Bahadoran Z, Mirmiran P, Hosseini-Esfahani F, et al. (2013) Fast food consumption and the risk of metabolic syndrome after 3-years of follow-up: Tehran Lipid and Glucose Study. Eur J Clin Nutr 67, 1303-1309. 
29. Hofmann SM, Dong HJ, Li Z, et al. (2002) Improved insulin sensitivity is associated with restricted intake of dietary glycoxidation products in the $\mathrm{db} / \mathrm{db}$ mouse. Diabetes 51, 2082-2089.

30. Peppa M, Goldberg T, Cai W, et al. (2002) Glycotoxins: a missing link in the "relationship of dietary fat and meat intake in relation to risk of type 2 diabetes in men". Diabetes Care 25, 1898-1899.

31. Lopez-Miranda J, Ordovas JM, Espino A, et al. (1994) Influence of mutation in human apolipoprotein A-1 gene promoter on plasma LDL cholesterol response to dietary fat. Lancet 343, 1246-1249.

32. Mata P, Lopez-Miranda J, Pocovi M, et al. (1998) Human apolipoprotein A-I gene promoter mutation influences plasma low density lipoprotein cholesterol response to dietary fat saturation. Atherosclerosis 137, 367-376.

33. Reedy J, Wirfalt E, Flood A, et al. (2010) Comparing 3 dietary pattern methods - cluster analysis, factor analysis, and index analysis - with colorectal cancer risk: The NIH-AARP Diet and Health Study. Am J Epidemiol 171, 479-487.
34. U.S. Department of Agriculture, U.S. Department of Health and Human Services (2010) Dietary Guidelines for Americans 2010. Washington, DC: U.S. Government Printing Office. http://www.dietaryguidelines.gov (accessed December 2010).

35. Gomez P, Perez-Martinez P \& Marin C (2010) APOA1 and $A P O A 4$ gene polymorphisms influence the effects of dietary fat on LDL particle size and oxidation in healthy young adults. J Nutr 140, 773-778.

36. Hargrove GM, Junco A \& Wong NC (1999) Hormonal regulation of apolipoprotein AI. J Mol Endocrinol 22, 103-111.

37. Lamon-Fava S, Ordovas JM \& Schaefer EJ (1999) Estrogen increases apolipoprotein (apo) A-I secretion in hep G2 cells by modulating transcription of the apo $A-I$ gene promoter. Arterioscler Thromb Vasc Biol 19, 2960-2965.

38. Asghari G, Rezazadeh A, Hosseini-Esfahani F, et al. (2012) Reliability, comparative validity and stability of dietary patterns derived from an FFQ in the Tehran Lipid and Glucose Study. Br J Nutr 108, 1109-1117. 